\title{
ON CLASSES OF EQUIVALENCE AND IDENTIFIABILITY OF AGE-DEPENDENT BRANCHING PROCESSES
}

\author{
RUI CHEN *** AND \\ OLLIVIER HYRIEN, ${ }^{* * * *}$ University of Rochester
}

\begin{abstract}
Age-dependent branching processes are increasingly used in analyses of biological data. Despite being central to most statistical procedures, the identifiability of these models has not been studied. In this paper we partition a family of age-dependent branching processes into equivalence classes over which the distribution of the population size remains identical. This result can be used to study identifiability of the offspring and lifespan distributions for parametric families of branching processes. For example, we identify classes of Markov processes that are not identifiable. We show that age-dependent processes with (nonexponential) gamma-distributed lifespans are identifiable and that Smith-Martin processes are not always identifiable.
\end{abstract}

Keywords: Identifiability; Bellman-Harris process; Sevastyanov process; Smith-Martin process

2010 Mathematics Subject Classification: Primary 60J80

Secondary $60 \mathrm{~J} 85$

\section{Introduction}

Let $Z(t)$ denote the size of a population governed by an age-dependent branching process started at $t=0$ with a single particle or cell of age 0 . Upon completion of its lifespan, every cell produces a random number of offspring $\xi \in \mathcal{g}=\{0,1,2, \ldots, J\}$, where $J$ is a given positive integer. Let $p:=\left(p_{0}, \ldots, p_{J}\right)$, where $p_{j}:=\mathbb{P}(\xi=j), j \in \mathcal{g}$, denote the offspring distribution. Set $h(u ; p):=\sum_{j \in \mathcal{g}} p_{j} u^{j}, u \in[-1,1]$, and $\mu:=\mathbb{E}(\xi)=\sum_{j \in \mathcal{g}} j p_{j}$ for its probability generating function (PGF) and expectation. A cell that produces a single offspring $(\xi=1)$ is said to be quiescent. This feature is relevant when modeling tumor growth ([1]; see also [5]). Throughout, we will implicitly assume that $p_{1} \in[0,1)$. Let $\mathcal{g}^{*}(p):=\left\{j \in \mathcal{g}: p_{j}>0\right\}$. For every $j \in \mathcal{g}^{*}(p)$, let $G_{j}(t):=\mathbb{P}(\tau \leq t \mid \xi=j), t \geq 0$, denote the conditional cumulative distribution function (CDF) of the lifespan $\tau$, given $\xi=j$. Write $\mathscr{D}$ for the class of all absolutely continuous (a.c.) CDFs $F$ that are proper and satisfy $F(0+)=0$ (the assumption of a.c. is not needed but simplifies the presentation). Assume that $G_{j} \in \mathscr{D}, j \in \mathscr{g}^{*}(p)$. As usual, every cell evolves independently of all other cells. Let $G=\left\{G_{j}, j \in \mathcal{g}^{*}(p)\right\}$. We will refer to $C=(p, G)$ as the characteristics of the process. The process is of Bellman-Harris type if the CDFs $G_{j}$ are identical for all $j \in \mathcal{g}^{*}(p)$. Otherwise, it allows the lifespan and offspring to be dependent, and belongs to the class of Sevastyanov processes [3], [4], [7].

Received 19 June 2013; revision received 17 September 2013.

* Postal address: Department of Biostatistics and Computational Biology, University of Rochester, Rochester, NY 14642, USA.

** Email address: rui_chen@urmc.rochester.edu

*** Email address: ollivier_hyrien@urmc.rochester.edu 
In this paper we address the following question: are there distinct characteristics $(p, G)$ under which the distribution of the process $Z(t)$ is identical? This question is relevant to the problem of model identifiability, which is a central prerequisite to most statistical procedures. Although age-dependent branching processes are widely used in biology, this question does not appear to have been studied for this class of models [2], [5], [9]. Answering this question will inform us about what can or cannot be estimated by only observing $Z(t)$, a situation that arises frequently in cell biology.

Let $\mathcal{P}$ denote the class of all processes that satisfy the above assumptions. It will be useful to define a subclass of processes included in $\mathcal{P}$, say $\mathcal{P}_{0}$, with characteristics $(p, G)$ satisfying $p_{1}=0$. We will say that two processes with characteristics $(p, G)$ and $(\hat{p}, \hat{G})$ are equivalent if, for all $t \geq 0$, the distribution of $Z(t)$ is the same under either characteristics. Let $\mathcal{C}_{p, G}$ denote the collection of processes included in $\mathcal{P}$ that are equivalent to the process with characteristics $(p, G)$. It forms an equivalence class, and our objective is to identify all the processes included in this class for any admissible set of characteristics $(p, G)$. If the class includes processes other than the process with characteristics $(p, G)$, then $p$ and $G$ cannot be unequivocally identified by the marginal distribution of $Z(t)$ for all $t \geq 0$.

We construct the class $\mathcal{C}_{p, G}$ in the next section. We proceed in three steps. First, we identify a collection of equivalent processes (Section 2.1). Next, by inverting the transformation that defines this collection about a properly chosen process, we find a larger collection of equivalent processes (Section 2.2). Finally, we prove, when $J=2$, which is typical of most biological applications, and $J=3$, that the larger collection is identical to $\mathcal{C}_{p, G}$ (Section 2.3). Each equivalence class contains a single process such that $p_{1}=0$. When $J=2$, the equivalence classes are fully characterized by the expectation and the variance of $Z(t)$ (Section 2.4). Our results are applicable to study identifiability of families of parametric models. For example, we find that the Markov version of the process is not always identifiable (Section 3.1). The age-dependent process with (nonexponential) gamma-distributed lifespan is identifiable (Section 3.2). We also find that the Smith-Martin process is not always identifiable (Section 3.3).

\section{Main results}

\subsection{A collection of equivalent processes}

For every $p_{1} \in[0,1)$ and $a \in\left[0, p_{1}\right]$, define $p^{(a)}=\left(p_{0}^{(a)}, \ldots, p_{J}^{(a)}\right)$, where

$$
p_{j}^{(a)}:= \begin{cases}\frac{p_{j}}{1-a}, & j \in \mathcal{g} \backslash\{1\}, \\ \frac{p_{1}-a}{1-a}, & j=1 .\end{cases}
$$

Note that $\mathscr{g}^{*}(p) \backslash\{1\}=\mathcal{G}^{*}\left(p^{(a)}\right) \backslash\{1\}$. By convention, when $p_{1}=0, G_{1}$ will denote any $\mathrm{CDF}$ in $\mathscr{D}$. For every $t \geq 0, j \in \mathfrak{g}^{*}(p)$, and $a \in\left[0, p_{1}\right]$, set

$$
G_{j}^{(a)}(t):=(1-a) G_{j} * \sum_{k=0}^{\infty} a^{k} G_{1}^{* k}(t),
$$

where $G_{j} * G_{1}(t):=\int_{0}^{t} G_{j}(t-x) \mathrm{d} G_{1}(x)$ denotes the convolution of $G_{j}$ and $F_{1}$, and $G_{1}^{* k}(t):=\int_{0}^{t} G_{1}^{*(k-1)}(t-x) \mathrm{d} G_{1}(x)$ is the $k$-fold convolution of $G_{1}$ with itself. For every $p_{1} \in[0,1)$ and $a \in\left[0, p_{1}\right]$, it can be verified that $G_{j}^{(a)}$ is the CDF of a proper distribution; it can be interpreted as the $\mathrm{CDF}$ of a (non-Markov) phase-type distribution, and the Laplace 
transform of $g_{j}^{(a)}(t):=\mathrm{d} G_{j}^{(a)}(t) / \mathrm{d} t$ is

$$
\mathcal{L}_{g_{j}^{(a)}}(s)=\frac{(1-a) \mathcal{L}_{g_{j}}(s)}{1-a \mathcal{L}_{g_{1}}(s)},
$$

where $\mathcal{L}_{g_{j}}$ is the Laplace transforms of $g_{j}(t):=\mathrm{d} G_{j}(t) / \mathrm{d} t$. Write $G^{(a)}=\left\{G_{j}^{(a)}, j \in\right.$ $\left.\mathcal{F}^{*}\left(p^{(a)}\right)\right\}$ and $C^{(a)}=\left(p^{(a)}, G^{(a)}\right)$.

Let $\delta_{p, G}$ denote the collection of processes with characteristics $\left(p^{(a)}, G^{(a)}\right), a \in\left[0, p_{1}\right]$. Since $\left(p^{(0)}, G^{(0)}\right)=(p, G), \wp_{p, G}$ includes the process with characteristics $(p, G)$. Thus, it is never empty. Moreover, since $p_{1}^{\left(p_{1}\right)}=0, \wp_{p, G}$ always includes at least one process from $\mathcal{P}_{0}$. This process will play a central role in constructing $\mathcal{C}_{p, G}$.

Theorem 1. For all $t \geq 0$, the distribution of the population size process $Z(t)$ is identical under all processes included in $\S_{p, G}$, that is, $\S_{p, G} \subseteq \mathcal{C}_{p, G}$.

Proof. Let $\Phi_{C}(u, t):=\mathbb{E}\left(u^{Z(t)} \mid Z(0)=1\right), u \in[-1,1]$ and $t \geq 0$, denote the PGF of $Z(t)$ under the process with characteristics $C$. Conditioning on the lifespan of the cell initiating the population yields

$$
\Phi_{C}(u, t)=u\left\{1-\sum_{j \in \mathcal{g}^{*}(p)} p_{j} G_{j}(t)\right\}+\sum_{j \in \mathcal{G}^{*}(p)} p_{j} \int_{0}^{t} \Phi_{C}(u, t-x)^{j} \mathrm{~d} G_{j}(x) .
$$

For every $j \in g$ and $u \in[-1,1]$, let $\mathscr{L}_{\Phi_{C}^{j}}(u, s):=\int_{0}^{\infty} \mathrm{e}^{-s t} \Phi_{C}(u, t)^{j} \mathrm{~d} t$ denote the Laplace transform of $\Phi_{C}(u, t)^{j}$. Put $\mathcal{L}_{\Phi_{C}}(u, t)=\mathcal{L}_{\Phi_{C}^{1}}(u, t)$. Since $\left|\Phi_{C}(u, t)\right| \leq 1$ for every $u \in$ $[-1,1]$ and $t \geq 0$, we have $\mathcal{L}_{\Phi_{C}^{j}}(u, s)<\infty$ for every $s>0$. Also, it follows from (3) that $\mathcal{L}_{\Phi_{C}}(u, s), s>0$, satisfies

$$
\mathcal{L}_{\Phi_{C}}(u, s)=\frac{u}{s}\left\{1-\sum_{j \in \mathcal{g}^{*}(p)} p_{j} \mathcal{L}_{g_{j}}(s)\right\}+\sum_{j \in \mathcal{g}^{*}(p)} p_{j} \mathcal{L}_{\Phi_{C}^{j}}(u, s) \mathcal{L}_{g_{j}}(s) .
$$

For every $a \in\left[0, p_{1}\right]$, (4) can be rearranged as

$$
\begin{aligned}
\left\{1-a \mathcal{L}_{g_{1}}(s)\right\} \mathcal{L}_{\Phi_{C}}(u, s)= & \frac{u}{s}\left\{1-\sum_{j \in \mathcal{g}^{*}(p) \backslash\{1\}} p_{j} \mathcal{L}_{g_{j}}(s)-p_{1} \mathcal{L}_{g_{1}}(s)\right\} \\
& +\sum_{j \in \mathcal{g}^{*}(p) \backslash\{1\}} p_{j} \mathcal{L}_{\Phi_{C}^{j}}(u, s) \mathcal{L}_{g_{j}}(s)+\left(p_{1}-a\right) \mathcal{L}_{\Phi_{C}}(u, s) \mathcal{L}_{g_{1}}(s) .
\end{aligned}
$$

Dividing both sides of the equation by $1-a \mathcal{L}_{g_{1}}(s)$ yields

$$
\begin{aligned}
\mathcal{L}_{\Phi_{C}}(u, s)= & \frac{u}{s}\left\{\frac{1}{1-a \mathcal{L}_{g_{1}}(s)}-\sum_{j \in \mathcal{g}^{*}\left(p^{(a)}\right) \backslash\{1\}} \frac{p_{j}}{1-a} \frac{(1-a) \mathcal{L}_{g_{j}}(s)}{1-a \mathcal{L}_{g_{1}}(s)}\right. \\
& \left.-\frac{p_{1}-a}{1-a} \frac{(1-a) \mathcal{L}_{g_{1}}(s)}{1-a \mathcal{L}_{g_{1}}(s)}-\frac{a \mathcal{L}_{g_{1}}(s)}{1-a \mathcal{L}_{g_{1}}(s)}\right\} \\
& +\sum_{j \in \mathcal{g}^{*}\left(p^{(a)}\right) \backslash\{1\}} \frac{p_{j}}{1-a} \frac{(1-a) \mathcal{L}_{g_{j}}(s)}{1-a \mathcal{L}_{g_{1}}(s)} \mathcal{L}_{\Phi_{C}^{j}}(u, s) \\
& +\frac{p_{1}-a}{1-a} \frac{(1-a) \mathcal{L}_{f_{1}}(s)}{1-a \mathcal{L}_{g_{1}}(s)} \mathcal{L}_{\Phi_{C}}(u, s) \\
= & \frac{u}{s}\left\{1-\sum_{j \in \mathcal{g}^{*}\left(p^{(a)}\right)} p_{j}^{(a)}(\theta) \mathcal{L}_{g_{j}^{(a)}}(s)\right\}+\sum_{j \in \mathcal{g}^{*}\left(p^{(a)}\right)} p_{j}^{(a)} \mathcal{L}_{\Phi_{C}^{j}}(u, s) \mathcal{L}_{g_{j}^{(a)}}(s) .
\end{aligned}
$$


By comparing (4) and (5), we deduce that $\mathcal{L}_{\Phi_{C}}(u, s)=\mathcal{L}_{\Phi_{C}(a)}(u, s)$; hence, the processes with characteristics $(p, G)$ and $\left(p^{(a)}, G^{(a)}\right), a \in\left[0, p_{1}\right]$, are equivalent.

\subsection{A larger collection of equivalent processes}

By inverting the transformation $(p, G) \rightarrow\left(p^{(a)}, G^{(a)}\right), a \in\left[0, p_{1}\right]$, about a properly chosen process in $\wp_{p, G}$, we will construct a collection of equivalent processes that is larger than $\wp_{p, G}$. Setting $a=p_{1}$ in (1) and (2) yields

$$
\begin{aligned}
p_{j}^{\left(p_{1}\right)} & = \begin{cases}\frac{p_{j}}{1-p_{1}}, & j \in \mathcal{g} \backslash\{1\}, \\
0, & j=1,\end{cases} \\
\mathcal{L}_{g_{j}^{\left(p_{1}\right)}}(s) & =\frac{\left(1-p_{1}\right) \mathcal{L}_{g_{j}}(s)}{1-p_{1} \mathcal{L}_{g_{1}}(s)}, \quad j \in \mathcal{F}^{*}(p) \backslash\{1\},
\end{aligned}
$$

which identifies a process in $\mathcal{P}_{0}$. We note that $\Phi_{C^{\left(p_{1}\right)}}(u, t)$ does not depend on $G_{1}^{\left(p_{1}\right)}$. Also, any process with characteristics $(\hat{p}, \hat{G})$ that satisfy

$$
\begin{gathered}
\hat{p}_{j}^{\left(\hat{p}_{1}\right)}=p_{j}^{\left(p_{1}\right)}, \quad j \in \mathcal{G} \backslash\{1\}, \\
\mathcal{L}_{\hat{g}_{j}^{\left(\hat{p}_{1}\right)}(s)=\mathcal{L}_{g_{j}^{\left(p_{1}\right)}}(s),} \quad j \in \mathcal{J}^{*}(p) \backslash\{1\},
\end{gathered}
$$

belongs to $\mathcal{C}_{p, G}$ because $\Phi_{\hat{C}}(u, t)=\Phi_{\hat{C}^{\left(\hat{p}_{1}\right)}}(u, t)=\Phi_{C^{\left(p_{1}\right)}}(u, t)=\Phi_{C}(u, t)$. By solving (6) we find that $(\hat{p}, \hat{G})$ satisfies

$$
\begin{gathered}
\hat{p}_{j}=p_{j}^{\left(p_{1}\right)}\left(1-\hat{p}_{1}\right), \quad j \in \mathcal{g} \backslash\{1\}, \\
\mathcal{L}_{\hat{g}_{j}}(s)=\mathcal{L}_{g_{j}}^{\left(p_{1}\right)}(s) \frac{\left\{1-\hat{p}_{1} \mathcal{L}_{\hat{g}_{1}}(s)\right\}}{1-\hat{p}_{1}}, \quad j \in \mathcal{G}^{*}(p) \backslash\{1\},
\end{gathered}
$$

where $\hat{p}_{1} \in[0,1)$ and $\hat{G}_{1} \in \mathcal{D}_{p, G}$, and $\mathscr{D}_{p, G} \subseteq \mathcal{D}$ is a set of distributions such that $\mathcal{L}_{\hat{g}_{j}}(s), j \in$ $\mathscr{g}^{*}(\hat{p}) \backslash\{1\}$, are the Laplace transforms of distributions in $\mathscr{D}$. Write $\left(p_{\hat{p}_{1}, \hat{G}_{1}}, G_{\hat{p}_{1}, \hat{G}_{1}}\right)$ for any characteristics that satisfy (7). Then, the collection of processes

$$
\left.\bar{s}_{p, G}:=\bigcup_{\hat{p}_{1} \in[0,1)} \bigcup_{\hat{G}_{1} \in \mathcal{D}_{p, G}} \text { \{process with characteristics }\left(p_{\hat{p}_{1}, \hat{G}_{1}}, G_{\hat{p}_{1}, \hat{G}_{1}}\right)\right\}
$$

is included in $\mathcal{C}_{p, G}$. It is also clear that $\S_{p, G} \subset \bar{\S}_{p, G}$.

\subsection{Exhaustivity of $\bar{\delta}_{p, G}$ when $J=2$ and $J=3$}

Our final step toward identifying $\mathcal{C}_{p, G}$ is to prove that it coincides with $\bar{\S}_{p, G}$. Denote by $\Phi_{C}^{(k)}(u, t):=\partial^{k} \Phi_{C}(u, t) / \partial u^{k}$ the $k$ th-order partial derivative of $\Phi_{C}(u, t), k=1,2, \ldots$ Let $m_{k}(t):=\mathbb{E}\left(\prod_{l=0}^{k-1}\{Z(t)-l\} \mid Z(0)=1\right), t \geq 0, k=1,2, \ldots$, denote the $k$ th-order factorial moment of $Z(t)$ under the process with characteristics $C$, and write $m(t)=m_{1}(t)$. We have $m_{k}(t)=\Phi^{(k)}(1, t)$. Differentiating both sides of (3) with respect to $u$ at $u=1$ yields the following integral equation for the expectation of the process:

$$
m(t)=1-\sum_{j \in \mathcal{G}^{*}(p)} p_{j} G_{j}(t)+\sum_{j \in \mathcal{I}^{*}(p)} j p_{j} \int_{0}^{t} m(t-x) \mathrm{d} G_{j}(x) .
$$


The second- and third-order factorial moments satisfy

$$
m_{2}(t)=\sum_{j \in \mathcal{g}^{*}(p)} j p_{j} \int_{0}^{t} m_{2}(t-x) \mathrm{d} G_{j}(x)+\sum_{j \in \mathcal{g}^{*}(p)} j(j-1) p_{j} \int_{0}^{t} m^{2}(t-x) \mathrm{d} G_{j}(x)
$$

and

$$
\begin{aligned}
m_{3}(t)= & \sum_{j \in \mathfrak{g}^{*}(p)} j p_{j} \int_{0}^{t} m_{3}(t-x) \mathrm{d} G_{j}(x) \\
& +\sum_{j \in \mathcal{g}^{*}(p)} 3 j(j-1) p_{j} \int_{0}^{t} m(t-x) m_{2}(t-x) \mathrm{d} G_{j}(x) \\
& +\sum_{j \in \mathcal{G}^{*}(p)} j(j-1)(j-2) p_{j} \int_{0}^{t} m(t-x)^{3} \mathrm{~d} G_{j}(x) .
\end{aligned}
$$

Let $\mathcal{L}_{m^{k}}(s)$ denote the Laplace transform of $m^{k}(t), k=1,2,3$. Taking the Laplace transform of both sides of (8)-(10) and rearranging the terms yields

$$
\begin{aligned}
\mathcal{L}_{m}(s) & =\frac{1-\sum_{j \in \mathfrak{I}^{*}(p)} p_{j} \mathcal{L}_{g_{j}}(s)}{s\left\{1-\sum_{j \in \mathfrak{I}^{*}(p)} j p_{j} \mathcal{L}_{g_{j}}(s)\right\}}, \\
\mathcal{L}_{m_{2}}(s) & =\frac{\mathcal{L}_{m^{2}}(s) \sum_{j \in \mathfrak{g}^{*}(p)} j(j-1) p_{j} \mathcal{L}_{g_{j}}(s)}{1-\sum_{j \in \mathcal{I}^{*}(p)} j p_{j} \mathcal{L}_{g_{j}}(s)},
\end{aligned}
$$

and

$$
\mathcal{L}_{m_{3}}(s)=\frac{\mathcal{L}_{m^{3}}(s) \sum_{j \in \mathcal{g}^{*}(p)} j(j-1)(j-2) p_{j} \mathcal{L}_{g_{j}}(s)}{1-\sum_{j \in \mathcal{g}^{*}(p)} j p_{j} \mathcal{L}_{g_{j}}(s)}+\frac{3 \mathcal{L}_{m m_{2}}(s) \mathcal{L}_{m_{2}}(s)}{\mathcal{L}_{m^{2}}(s)},
$$

where $\mathcal{L}_{m m_{2}}(s)$ denotes the Laplace transform of $m(t) m_{2}(t)$.

Lemma 1. Suppose that $J=2$ or $J=3$. For every admissible ( $p, G)$, the equivalence class $\mathcal{C}_{p, G}$ includes a single process in $\mathcal{P}_{0}$.

Proof. Assume first that $J=3$. Consider two processes in $\mathcal{P}_{0}$ with characteristics $C=$ $(p, G)$ and $\hat{C}=(\hat{p}, \hat{G})$. Thus, $p_{1}=0$ and $\hat{p}_{1}=0$. Suppose that these processes are equivalent, that is, they both belong to $\mathcal{C}_{p, G}$. Then $\Phi_{C}(u, t)=\Phi_{\hat{C}}(u, t)$ and $\Phi_{C}^{(k)}(1, t)=\Phi_{\hat{C}}^{(k)}(1, t), u \in$ $[-1,1], t \geq 0$, and $k=1,2,3$. Write $\hat{m}_{k}(t)$ for the $k$ th-order factorial moment of the process with characteristics $\hat{C}$. Hence, $\mathcal{L}_{\Phi_{C}^{(k)}}(s)=\mathcal{L}_{\Phi_{\hat{C}}^{(k)}}(s)$, which, using identities (11)-(13), yields

$$
\begin{aligned}
\frac{1-p_{0} \mathcal{L}_{g_{0}}(s)-p_{2} \mathcal{L}_{g_{2}}(s)-p_{3} \mathcal{L}_{g_{3}}(s)}{d(s)} & =\frac{1-\hat{p}_{0} \mathcal{L}_{\hat{g}_{0}}(s)-\hat{p}_{2} \mathcal{L}_{\hat{g}_{2}}(s)-\hat{p}_{3} \mathcal{L}_{\hat{g}_{3}}(s)}{\hat{d}(s)}, \\
\frac{\mathcal{L}_{m^{2}}(s)\left\{2 p_{2} \mathcal{L}_{g_{2}}(s)+6 p_{3} \mathcal{L}_{g_{3}}(s)\right\}}{d(s)} & =\frac{\mathcal{L}_{m^{2}}(s ; \hat{C})\left\{2 \hat{p}_{2} \mathcal{L}_{\hat{g}_{2}}(s)+6 \hat{p}_{3} \mathcal{L}_{\hat{g}_{3}}(s)\right\}}{\hat{d}(s)}, \\
\frac{6 \mathcal{L}_{m^{3}}(s) p_{3} \mathcal{L}_{g_{3}}(s)}{d(s)}+\frac{3 \mathcal{L}_{m m_{2}}(s) \mathcal{L}_{m_{2}}(s)}{\mathcal{L}_{m^{2}}(s)} & =\frac{6 \mathcal{L}_{\hat{m}^{3}}(s) \hat{p}_{3} \mathcal{L}_{\hat{g}_{3}}(s)}{\hat{d}(s)}+\frac{3 \mathcal{L}_{\hat{m} \hat{m}_{2}}(s ;) \mathcal{L}_{\hat{m}_{2}}(s)}{\mathcal{L}_{\hat{m}^{2}}(s)},
\end{aligned}
$$


where $d(s)=1-2 p_{2} \mathcal{L}_{g_{2}}(s)-3 p_{3} \mathcal{L}_{g_{3}}(s)$ and $\hat{d}(s)=1-2 \hat{p}_{2} \mathcal{L}_{\hat{g}_{2}}(s)-3 \hat{p}_{3} \mathcal{L}_{\hat{g}_{3}}(s)$. Since $\mathcal{L}_{m^{k}}(s)=\mathcal{L}_{\hat{m}^{k}}(s), k=1,2,3$, and $\mathcal{L}_{m m_{2}}(s)=\mathcal{L}_{\hat{m} \hat{m}_{2}}(s)$, the above system reduces to

$$
\frac{p_{j} \mathcal{L}_{g_{j}}(s)}{d(s)}=\frac{\hat{p}_{j} \mathcal{L}_{\hat{g}_{j}}(s)}{\hat{d}(s)}, \quad j=0,2,3 .
$$

The above equations obtained when $j=2,3$ yield

$$
\begin{aligned}
& p_{2} \mathcal{L}_{g_{2}}(s)-3 p_{2} \mathcal{L}_{g_{2}}(s) \hat{p}_{3} \mathcal{L}_{\hat{g}_{3}}(s)=\hat{p}_{2} \mathcal{L}_{\hat{g}_{2}}(s)-3 \hat{p}_{2} \mathcal{L}_{\hat{g}_{2}}(s) p_{3} \mathcal{L}_{g_{3}}(s), \\
& p_{3} \mathcal{L}_{g_{3}}(s)-2 p_{3} \mathcal{L}_{g_{3}}(s) \hat{p}_{2} \mathcal{L}_{\hat{g}_{2}}(s)=\hat{p}_{3} \mathcal{L}_{\hat{g}_{3}}(s)-2 \hat{p}_{3} \mathcal{L}_{\hat{g}_{3}}(s) p_{2} \mathcal{L}_{g_{2}}(s),
\end{aligned}
$$

which imply that $2 p_{2} \mathcal{L}_{g_{2}}(s)+3 p_{3} \mathcal{L}_{g_{3}}(s)=2 \hat{p}_{2} \mathcal{L}_{\hat{g}_{2}}(s)+3 \hat{p}_{3} \mathcal{L}_{\hat{g}_{3}}(s)$; hence, $d(s)=\hat{d}(s)$, and the system of equations (14) reduces to $p_{j} \mathcal{L}_{g_{j}}(s)=\hat{p}_{j} \mathcal{L}_{\hat{g}_{j}}(s), j=0,2,3$. Hence, $(\hat{p}, \hat{G})=(p, G)$ since the distributions $G_{j}$ and $\hat{G}_{j}, j \in \mathcal{g}^{*}(p)$, are all proper. This completes the proof when $J=3$. The case $J=2$ is treated similarly except that we only use the first and second equations of the system (14), and we set $p_{3}=\hat{p}_{3}=0$.

Theorem 2. We have $\bar{\delta}_{p, G}=\mathcal{C}_{p, G}$ for every admissible $(p, G)$ when $J=2$ and $J=3$.

Proof. We already know that $\bar{\delta}_{p, G} \subseteq \mathcal{C}_{p, G}$. To prove that the converse holds, let $(\hat{p}, \hat{G})$ denote the characteristics of any process included in $\mathcal{C}_{p, G}$. Then, by construction, the process with characteristics $\left(\hat{p}^{\left(\hat{p}_{1}\right)}, \hat{G}^{\left(\hat{p}_{1}\right)}\right)$ belongs to $\mathcal{P}_{0}$. We also know from Lemma 1 that $\left(\hat{p}^{\left(\hat{p}_{1}\right)}, \hat{G}^{\left(\hat{p}_{1}\right)}\right)=\left(p^{\left(p_{1}\right)}, G^{\left(p_{1}\right)}\right)$. Hence, the process with characteristics $(\hat{p}, \hat{G})$ belongs to $\bar{\delta}_{p, G}$, which implies that $\mathcal{C}_{p, G} \subseteq \bar{\delta}_{p, G}$. This completes the proof.

\subsection{Characterization of $e_{p, G}$ using moments when $J=2$}

In data analyses, model parameters are sometimes estimated using moments of the process rather than its distribution. Then, a relevant question is: which moments are sufficient to fully characterize the equivalence class $\mathcal{C}_{p, G}$ ? We show below that the answer is simply the expectation and variance when $J=2$. This property does not appear to generalize when $J>2$, however.

Theorem 3. Assume that $J=2$ and that the marginal distribution of $\{Z(t), t \geq 0\}$ is determined by its moments. Then $\mathcal{C}_{p, G}=\{$ processes with characteristics $(\hat{p}, \hat{G}): \hat{m}(t)=$ $\left.m(t), \hat{m}_{2}(t)=m_{2}(t), t \geq 0\right\}$.

Proof. To simplify the presentation, we assume, when $p_{j}=0$, that $G_{j}$ is any arbitrary CDF in $\mathscr{D}$. For $k=2,3, \ldots$, it can be shown by induction and using the identity $m_{k}(t)=\Phi_{C}^{(k)}(1, t)$ that

$$
m_{k}(t)=p_{2} \int_{0}^{t} \sum_{r=1}^{\lfloor k / 2\rfloor} c_{k r} m_{r}(t-x) m_{k-r}(t-x) \mathrm{d} G_{2}(x)+\sum_{j=1}^{2} j p_{j} \int_{0}^{t} m_{k}(t-x) \mathrm{d} G_{j}(x),
$$

where $\lfloor k / 2\rfloor$ denotes the largest integer less than or equal to $k / 2$, and $c_{k r}$ are some positive integers. Then

$$
\mathcal{L}_{m_{k}}(s)=p_{2} l_{k}(s) \mathcal{L}_{g_{2}}(s)+\mathcal{L}_{m_{k}}(s) \sum_{j=1}^{2} j p_{j} \mathcal{L}_{g_{j}}(s),
$$


where $l_{k}(s)$ is the Laplace transform of $\sum_{r=1}^{\lfloor k / 2\rfloor} c_{k r} m_{r}(t) m_{k-r}(t)$. Hence,

$$
\mathcal{L}_{m_{k}}(s)=\frac{l_{k}(s) p_{2} \mathcal{L}_{g_{2}}(s)}{1-\sum_{j=1}^{2} j p_{j} \tilde{\mathcal{L}}_{g_{j}}(s)} .
$$

Let $\hat{C}=(\hat{p}, \hat{G})$ denote the characteristics of any process in $\mathcal{C}_{p, G}$. Then $\Phi_{\hat{C}}(u, t)=\Phi_{C}(u, t)$, $t \geq 0, u \in[-1,1]$. By assumption, $\Phi_{c}(u, t)$ is determined by its moments. Hence, $\mathcal{C}_{p, G}=$ \{processes with characteristics $\left.(\hat{p}, \hat{G}): \hat{m}_{k}(t)=m_{k}(t), t \geq 0, k \in \mathbb{N}\right\}$, where $\mathbb{N}=\{1,2, \ldots\}$. We note that $\hat{m}_{k}(t)=m_{k}(t)$ implies that $\mathcal{L}_{\hat{m}_{k}}(s)=\mathcal{L}_{m_{k}}(s)$ and $\hat{l}_{k}(s)=l_{k}(s), k \in \mathbb{N}$, from which we deduce, when $k=2$, that

$$
\frac{p_{2} \mathcal{L}_{g_{2}}(s)}{1-\sum_{j=1}^{2} j p_{j} \mathcal{L}_{g_{j}}(s)}=\frac{\hat{p}_{2} \mathcal{L}_{\hat{g}_{2}}(s)}{1-\sum_{j=1}^{2} j \hat{p}_{j} \mathcal{L}_{\hat{g}_{j}}(s)},
$$

and, when $k=3,4, \ldots$, that

$$
\frac{l_{k}(s) p_{2} \mathcal{L}_{g_{2}}(s)}{1-\sum_{j=1}^{2} j p_{j} \mathcal{L}_{g_{j}}(s)}=\frac{l_{k}(s) \hat{p}_{2} \mathcal{L}_{\hat{g}_{2}}(s)}{1-\sum_{j=1}^{2} j \hat{p}_{j} \mathcal{L}_{\hat{g}_{j}}(s)} .
$$

Equation (16) and (17) are clearly equivalent when $l_{k}(s) \neq 0$. When $l_{k}(s)=0$, we deduce from (15) that $\mathcal{L}_{m_{k}}(s)=0$ and $m_{k}(t)=0, k=3,4, \ldots$. Thus, in either case, we conclude that $\mathcal{C}_{p, G}=$ \{processes with characteristics $\left.(\hat{p}, \hat{G}): \hat{m}_{k}(t)=m_{k}(t), t \geq 0, k=1,2\right\}$, which completes the proof.

\section{Application to model identifiability}

The results obtained in Section 2 are applicable to study identifiability of branching processes when specific parametric assumptions are made about the lifespan distributions. To shorten the discussion, we only consider the case in which $J=2$.

\subsection{Exponentially distributed lifespan}

We assume here that $\tau$ is conditionally exponentially distributed, given $\{\xi=j\}: G_{j}(t)=$ $1-\mathrm{e}^{-\psi_{j} t}, t \geq 0$, for some $\psi_{j} \in \mathbb{R}_{+}^{*}, j \in \mathcal{g}^{*}(p)$. The resulting class of processes is denoted by $\mathcal{M}$. We note that $\mathcal{L}_{g_{j}}(s)=\psi_{j} /\left(\psi_{j}+s\right), j \in \mathcal{g}^{*}(p)$. It is defined for $s \in\left(-\psi_{j}, \infty\right)$, and extendable to $s \in\left(-\infty,-\psi_{j}\right) \cup\left(-\psi_{j}, \infty\right)$ by analytic continuation.

For every admissible $(p, G)$, let $\mathcal{C}_{p, G}^{\mathcal{M}}=\mathcal{C}_{p, G} \cap \mathcal{M}$ denote the class of all processes included in $\mathcal{M}$ that are equivalent to the process with characteristics $(p, G)$. We say that the characteristics $(p, G)$ are identified by $\{Z(t), t \geq 0\}$ if and only if $\mathcal{C}_{p, G}^{\mathcal{M}}$ includes only the process with characteristics $(p, G)$. To establish identifiability of $(p, G)$, or lack thereof, it suffices to construct the class $\mathcal{C}_{p, G}^{\mathcal{M}}$. Let $(\hat{p}, \hat{G})$ denote the characteristics of any process in $\mathcal{C}_{p, G}^{\mathcal{M}}$. Then $\hat{G}_{j}, j \in g^{*}(\hat{p})$, is exponential.

Assume first that $p_{1}=0$. If $\hat{p}_{1}=0$, Lemma 1 implies that $(\hat{p}, \hat{G})=(p, G)$. If $\hat{p}_{1} \in(0,1)$, we know from Theorem 2 and (7) that, for every $j \in \mathcal{G}^{*}(p) \backslash\{1\}$,

$$
\frac{\left(1-\hat{p}_{1}\right) \hat{\psi}_{j}}{\hat{\psi}_{j}+s}=\frac{\psi_{j}}{\psi_{j}+s}\left(1-\frac{\hat{p}_{1} \hat{\psi}_{1}}{\hat{\psi}_{1}+s}\right)
$$

Rearranging the terms in the above identity leads to the polynomial equation

$$
\left(1-\hat{p}_{1}\right) \hat{\psi}_{j}\left(\psi_{j}+s\right)\left(\hat{\psi}_{1}+s\right)=\psi_{j}\left\{\left(1-\hat{p}_{1}\right) \hat{\psi}_{1}+s\right\}\left(\hat{\psi}_{j}+s\right) .
$$


This identity holds if and only if $\hat{\psi}_{j}=\hat{\psi}_{1}=\psi_{j} /\left(1-\hat{p}_{1}\right)$. Hence, for any $j_{1}, j_{2} \in \mathcal{g}^{*}(p)$, $\psi_{j_{1}}=\psi_{j_{2}}$, and the process with the characteristics $(p, G)$ must be Bellman-Harris. Write $\psi:=\psi_{j}, j \in g^{*}(p)$, and we have $\hat{\psi}_{j}=\psi /\left(1-\hat{p}_{1}\right)$. Using the first equation in (7), we deduce that $\hat{p}_{j}=p_{j}\left(1-\hat{p}_{1}\right), \hat{p}_{1} \in(0,1)$.

Assume next that $p_{1} \in(0,1)$. If $\hat{p}_{1}=0$, a similar line of arguments shows that the process with characteristics $(\hat{p}, \hat{G})$ satisfying $\hat{\psi}_{j}=\psi\left(1-p_{1}\right), \hat{p}_{j}=p_{j} /\left(1-p_{1}\right), j \in \mathcal{g}^{*}(p)\{1\}$, belongs to $\mathcal{C}_{p, G}^{\mathcal{M}}$ if $\psi_{j}=\psi, j \in \mathcal{g}^{*}(p)$.

Now assume that $p_{1} \in(0,1)$ and $\hat{p}_{1} \in(0,1)$. Then, for every $j \in \mathcal{g}^{*}(p)$, we have

$$
\frac{\left(1-\hat{p}_{1}\right) \hat{\psi}_{j}}{\hat{\psi}_{j}+s}=\frac{\left(1-p_{1}\right) \psi_{j}}{\psi_{j}+s}\left(1-\frac{\hat{p}_{1} \hat{\psi}_{1}}{\hat{\psi}_{1}+s}\right) /\left(1-\frac{p_{1} \psi_{1}}{\psi_{1}+s}\right) .
$$

Rearranging the terms in the above identity leads to the polynomial equation

$$
\left(1-\hat{p}_{1}\right) \hat{\psi}_{j}\left\{\left(1-p_{1}\right) \psi_{1}+s\right\}\left(\psi_{j}+s\right)\left(\hat{\psi}_{1}+s\right)=\left(1-p_{1}\right) \psi_{j}\left\{\left(1-\hat{p}_{1}\right) \hat{\psi}_{1}+s\right\}\left(\hat{\psi}_{j}+s\right)\left(\psi_{1}+s\right) .
$$

Solving this equation together with the first equation in (7) for each $j \in \mathcal{G}^{*}(p) \backslash\{1\}$ separately leads to three admissible sets of equations, denoted by $A_{j}, B_{j}$, and $C_{j}$ (indexed by $j$ ):

$$
\left(A_{j}\right)\left\{\begin{array} { l } 
{ ( 1 - \hat { p } _ { 1 } ) \hat { \psi } _ { j } = ( 1 - p _ { 1 } ) \psi _ { j } , } \\
{ \hat { \psi } _ { 1 } = \psi _ { 1 } , } \\
{ \hat { \psi } _ { j } = \psi _ { j } , } \\
{ ( 1 - \hat { p } _ { 1 } ) \hat { \psi } _ { 1 } = ( 1 - p _ { 1 } ) \psi _ { 1 } , } \\
{ \frac { \hat { p } _ { j } } { 1 - \hat { p } _ { 1 } } = \frac { p _ { j } } { 1 - p _ { 1 } } , }
\end{array} \quad ( B _ { j } ) \left\{\begin{array}{l}
\left(1-\hat{p}_{1}\right) \hat{\psi}_{j}=\left(1-p_{1}\right) \psi_{j}, \\
\psi_{1}=\psi_{j}, \\
\hat{\psi}_{1}=\hat{\psi}_{2}, \\
\left(1-p_{1}\right) \psi_{j}=\left(1-\hat{p}_{1}\right) \hat{\psi}_{j}, \\
\frac{\hat{p}_{j}}{1-\hat{p}_{1}}=\frac{p_{j}}{1-p_{1}},
\end{array}\right.\right.
$$

and

$$
\left(C_{j}\right)\left\{\begin{array}{l}
\left(1-\hat{p}_{1}\right) \hat{\psi}_{2}=\left(1-p_{1}\right) \psi_{2} \\
\hat{\psi}_{1}=\psi_{1} \\
\psi_{2}=\left(1-\hat{p}_{1}\right) \hat{\psi}_{1} \\
\hat{\psi}_{2}=\left(1-p_{1}\right) \psi_{1} \\
\frac{\hat{p}_{j}}{1-\hat{p}_{1}}=\frac{p_{j}}{1-p_{1}}
\end{array}\right.
$$

Assume first that $p_{0} p_{2}>0$. Then the collection of Markov processes that are equivalent to the process with characteristics $(p, G)$ is determined by simultaneously solving the equations $X_{0}$ and $Y_{2}$, where $X$ and $Y$ stand symbolically for either $A, B$, or $C$. There are nine such combinations.

- For equations $A_{0}$ and $A_{2}$, it is easy to show that the only solution is $(\hat{p}, \hat{G})=(p, G)$. Thus, here $(\hat{p}, \hat{G})$ identifies the process with characteristics $(p, G)$.

- Equations $B_{0}$ and $B_{2}$ admit solutions if and only if $\psi_{j}=\psi, j \in g^{*}(p)$, that is, the process with characteristics $(p, G)$ must be Bellman-Harris. When this condition is met, the solutions to the two equations satisfy $\hat{\psi}_{j}=\hat{\psi}, j \in g^{*}(p)$, where $\hat{\psi}=\psi(1-$ $\left.p_{1}\right) /\left(1-\hat{p}_{1}\right), \hat{p}_{j}=p_{j}\left(1-\hat{p}_{1}\right) /\left(1-p_{1}\right)$, and $\hat{p}_{1} \in(0,1)$. Thus, any Markov BellmanHarris process admits infinitely many equivalent processes in $\mathcal{M}$, which are also all (Markov) Bellman-Harris. 
- Equations $C_{0}$ and $C_{2}$ admit solutions if and only if $\psi_{0}=\psi_{2}$ and $\psi_{0} \leq \psi_{1}$. Under these constraints, the unique solution to the two sets of equations is $\hat{\psi}_{1}=\psi_{1}, \hat{\psi}_{0}=\hat{\psi}_{2}=$ $\left(1-p_{1}\right) \psi_{1}, \hat{p}_{1}=1-\psi_{0} / \psi_{1}$, and $\hat{p}_{j}=p_{j} \psi_{0} /\left\{\left(1-p_{1}\right) \psi_{1}\right\}, j=0,2$. This solution always differs from $(p, G)$, except when $p_{1}=1-\psi_{0} / \psi_{1}$. Thus, processes that satisfy the conditions $\psi_{0}=\psi_{2}$ and $\psi_{0} \leq \psi_{1}$ are identifiable only if $p_{1}=1-\psi_{0} / \psi_{1}$. Otherwise, there exists a unique $(\hat{p}, \hat{G})$ that differs from $(p, G)$ under which the distribution of the process $Z(t)$ does not change.

- Any other pair of equations admits solutions only under specific restrictions on $(p, G)$. For example, equations $A_{0}$ and $B_{2}$ will have a solution provided that $\psi_{1}=\psi_{2}$. When the conditions are met, the only solution to the equations is $(\hat{p}, \hat{G})=(p, G)$, and, therefore, identifies the initial process.

When either $p_{0}=0$ or $p_{2}=0$, we obtain the same set of solutions as above (details are omitted). We summarize the above findings in the following corollary.

Corollary 1. Suppose that $J=2$ and, for every $j \in \mathcal{G}^{*}(p)$, that $G_{j}(t)=1-\mathrm{e}^{-\psi_{j} t}, t \geq 0$, for some $\psi_{j} \in \mathbb{R}_{+}^{*}$. Then $(p, G)$ is uniquely identified by the process $\{Z(t), t \geq 0\}$, except in the following cases.

Case 1. If $\psi_{j}=\psi, j \in \mathcal{g}^{*}(p)$ (Bellman-Harris case), then $\mathcal{C}_{p, G}^{\mathcal{M}}$ includes the Markov processes with characteristics $(\hat{p}, \hat{G}) \in\left\{\hat{p}_{1} \in[0,1), \hat{p}_{j}=p_{j}\left(1-\hat{p}_{1}\right) /\left(1-p_{1}\right), j \in\right.$ $\left.\mathcal{g}^{*}(p) \backslash\{1\}, \hat{\psi}_{j}=\psi\left(1-p_{1}\right) /\left(1-\hat{p}_{1}\right), j \in \mathcal{g}^{*}(\hat{p})\right\}$.

Case 2. If $\psi_{j}=\psi, j \in \mathcal{G}^{*}(p) \backslash\{1\}, p_{1} \in(0,1), \psi<\psi_{1}$, and $p_{1} \neq 1-\psi / \psi_{1}$ ('extended' Bellman-Harris case), then $\mathcal{C}_{p, G}^{\mathcal{M}}$ consists of the process in $\mathcal{M}$ with characteristics $(p, G)$ and $(\hat{p}, \hat{G})$, where $\hat{p}_{1} \stackrel{p, G}{=} 1-\psi / \psi_{1}, \hat{p}_{j}=p_{j} \psi /\left\{\left(1-p_{1}\right) \psi_{1}\right\}, \hat{\psi}_{1}=\psi_{1}$, and $\hat{\psi}_{0}=\hat{\psi}_{2}=\left(1-p_{1}\right) \psi_{1}, j \in \mathcal{g}^{*}(p) \backslash\{1\}$.

Remark 1. Corollary 1 identifies two classes of processes in $\mathcal{M}$ that are not identifiable. The characteristics of the equivalent processes differ widely over $\mathcal{C}_{p, G}$ when $(p, G)$ identifies a Bellman-Harris process. As an illustration, consider the Markov process with offspring distribution $p=\left(\frac{1}{5}, \frac{1}{2}, \frac{3}{10}\right)$ and exponentially distributed lifespan with parameters $\psi_{0}=\psi_{1}=$ $\psi_{2}=1$. This process is of Bellman-Harris type. The class of processes in $\mathcal{M}$ equivalent to this process is determined by case 1 of Corollary 1 , and it includes the processes with offspring distributions $\hat{p}=\left[\frac{2}{5}\left(1-\hat{p}_{1}\right), \hat{p}_{1}, \frac{3}{5}\left(1-\hat{p}_{1}\right)\right]$ and exponentially distributed lifespan with parameters $\hat{\psi}_{0}=\hat{\psi}_{1}=\hat{\psi}_{2}=1 / 2\left(1-\hat{p}_{1}\right)$, where $\hat{p}_{1} \in[0,1)$. In particular, if $\hat{p}_{1}=0$, we obtain the process parameterized by $\hat{p}=\left(\frac{2}{5}, 0, \frac{3}{5}\right)$ and $\hat{\psi}_{0}=\hat{\psi}_{2}=0.5$, which belongs to $\mathcal{P}_{0}$. In Figure 1 (a) we present examples of probability density functions $\hat{g}_{2}$ for a sample of processes that belong to the equivalence class. In Figure 1(b)-(c) we plot the set of probability density functions $\hat{g}_{2}$ when the Bellman-Harris structure of the process is relaxed. For example, in Figure 1(b) we set $\psi_{1}=1.5$ (all other parameter values are identical to those used in Figure 1(a)), and find using case 2 of Corollary 1 that the class of equivalent processes in $\mathcal{M}$ includes a second process with offspring distribution $\hat{p}=\left(\frac{4}{15}, \frac{1}{3}, \frac{2}{5}\right)$ and exponentially distributed lifespan with parameters $\hat{\psi}_{1}=1.5$ and $\hat{\psi}_{0}=\hat{\psi}_{2}=0.75$. In Figure 1(c) we set $\psi_{1}=0.5$, while in Figure $1(\mathrm{~d})$ we set $\psi_{1}=1$ and $\psi_{0}=2$. In these two cases, the class of equivalent processes includes only the original process, which is therefore identifiable.

Remark 2. From a statistical standpoint, when $Z(t)$ is observed at discrete time points, the likelihood function can be solely expressed using the marginal distribution of $\{Z(t), t \geq 0\}$, and the model parameters are therefore not always identifiable. The maximum likelihood estimator 

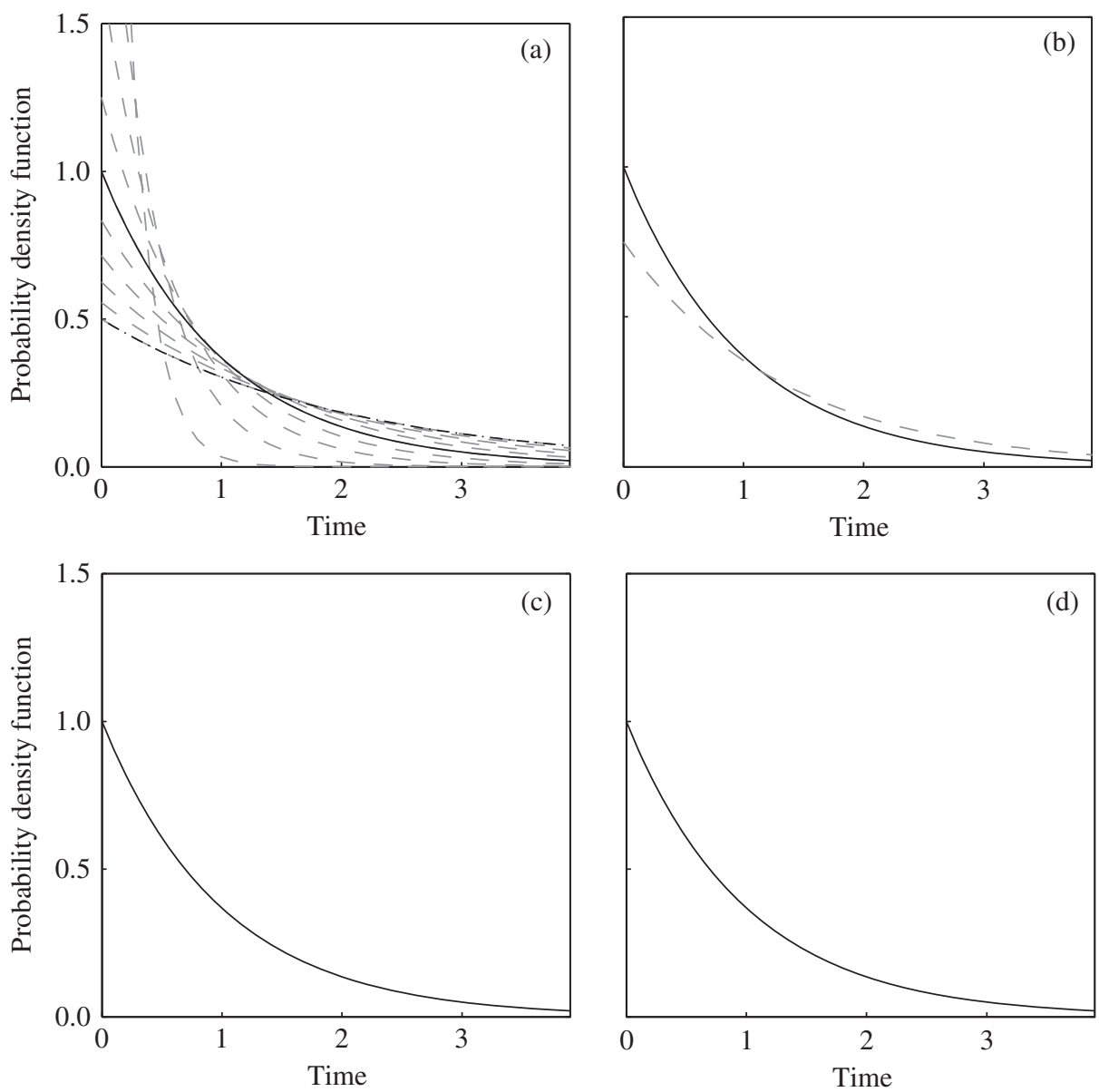

FiguRE 1: Representation of the set of probability density functions $\hat{g}_{2}$ over the class of equivalent processes for four processes in $\mathcal{M}$ : (a) $\psi_{0}=\psi_{1}=\psi_{2}=1$ (Bellman-Harris process); (b) $\psi_{0}=\psi_{2}=1$ and $\psi_{1}=1.5\left(>\psi_{0}\right.$ and $\left.>\psi_{2}\right)$ ('extended' Bellman-Harris process); (c) $\psi_{0}=\psi_{2}=1$ and $\psi_{1}<1$ ('extended' Bellman-Harris process); (d) $\psi_{0} \neq \psi_{2}, \psi_{1}>0$, and $\psi_{2}=1$. We set $p_{1}=\frac{1}{2}$ in all cases. Each plot shows $g_{2}$ and $\hat{g}_{2}$ (whenever there exists $\hat{g}_{2} \neq g_{2}$ ) for the process with characteristics $(p, G)$ (solid lines), representative processes of the equivalence class (dash-dot line), and the equivalent processes in $\mathcal{M}$ in $\mathcal{P}_{0}$ (dashed lines). The model is nonidentifiable in cases (a) and (b), and identifiable in cases (c) and (d).

is not consistent, at least in the traditional sense [6]. If modeling quiescence is not of primary interest, this nonidentifiability issue may be avoided by imposing $\hat{p}_{1}=p_{1}=0$. Under such a restriction, the interpretation of $G_{j}, j \in \mathcal{g}^{*}(p)$, may change because the time to producing $j$ offspring could now include a resting phase latently embedded in the lifespan.

\subsection{Gamma-distributed lifespan}

We now extend the Markov process by assuming that the lifespan is gamma distributed:

$$
G_{j}(t):=\int_{0}^{t} \frac{\kappa_{j}^{\omega_{j}}}{\Gamma\left(\omega_{j}\right)} x^{\omega_{j}-1} \mathrm{e}^{-\kappa_{j} x} \mathrm{~d} x
$$


for some $\psi_{j}:=\left(\omega_{j}, \kappa_{j}\right) \in \mathbb{R}_{+}^{*} \times \mathbb{R}_{+}^{*}, j \in \mathcal{g}^{*}(p)$. We have $\mathcal{L}_{g_{j}}(s)=\kappa_{j}^{\omega_{j}} /\left(\kappa_{j}+s\right)^{\omega_{j}}$, defined for $s \in\left(-\kappa_{j}, \infty\right)$, but also extendable to $s \in\left(-\infty,-\kappa_{j}\right) \cup\left(-\kappa_{j}, \infty\right)$ by analytic continuation. The assumption of a gamma-distributed lifespan is frequently made in practice [3], [9]. We obtain the Markov process of the previous section if $\omega_{j}=1, j \in \mathcal{g}^{*}(p)$. In the following result we show, when $\omega_{j} \neq 1$, that it is identifiable.

Corollary 2. Suppose that $J=2$ and, for every $j \in \mathcal{g}^{*}(p)$, that $G_{j}$ is a gamma distribution with parameters $\kappa_{j}>0, \omega_{j}>0$, and $\omega_{j} \neq 1$. Then $(p, G)$ is uniquely identified by the process $\{Z(t), t \geq 0\}$.

Proof. Let $(\hat{p}, \hat{G})$ denote the characteristics of any process included in $\mathcal{C}_{p, G}$. Assume first that $p_{1}=0$. If $\hat{p}_{1}=0$, Lemma 1 implies that $(\hat{p}, \hat{G})=(p, G)$. If $\hat{p}_{1} \in(0,1),(6)$ gives

$$
\left(1-\hat{p}_{1}\right)\left(\frac{\hat{\kappa}_{j}}{\hat{\kappa}_{j}+s}\right)^{\hat{\omega}_{j}}=\left(\frac{\kappa_{j}}{\kappa_{j}+s}\right)^{\omega_{j}}\left\{1-\hat{p}_{1}\left(\frac{\hat{\kappa}_{1}}{\hat{\kappa}_{1}+s}\right)^{\hat{\omega}_{1}}\right\} .
$$

Rearranging the terms in the above identity leads to the equation

$$
\left(1-\hat{p}_{1}\right) \hat{\kappa}_{j}^{\hat{\omega}_{j}}\left(\hat{\kappa}_{1}+s\right)^{\hat{\omega}_{1}}\left(\kappa_{j}+s\right)^{\omega_{j}}=\kappa_{j}^{\omega_{j}}\left(\hat{\kappa}_{j}+s\right)^{\hat{\omega}_{j}}\left\{\left(\hat{\kappa}_{1}+s\right)^{\hat{\omega}_{1}}-\hat{p}_{1} \hat{\kappa}_{1}^{\hat{\omega}_{1}}\right\} .
$$

Dividing both sides of (20) by $\left(\hat{\kappa}_{1}+s\right)^{\hat{\omega}_{1}}\left(\kappa_{j}+s\right)^{\omega_{j}}$ and letting $s \rightarrow \infty$ yields

$$
\left(1-\hat{p}_{1}\right) \hat{\kappa}_{j}^{\hat{\omega}_{j}}=\kappa_{j}^{\omega_{j}} \lim _{s \rightarrow \infty}\left\{\frac{\left(\hat{\kappa}_{j}+s\right)^{\hat{\omega}_{j}}}{\left(\kappa_{j}+s\right)^{\omega_{j}}}-\frac{\hat{p}_{1} \hat{\kappa}_{1}^{\hat{\omega}_{1}}\left(\hat{\kappa}_{j}+s\right)^{\hat{\omega}_{j}}}{\left(\kappa_{j}+s\right)^{\omega_{j}}\left(\hat{\kappa}_{1}+s\right)^{\hat{\omega}_{1}}}\right\} .
$$

In order for the right-hand side to converge to a constant, we must have $\hat{\omega}_{j}=\omega_{j}$, which implies that $\left(1-\hat{p}_{1}\right) \hat{\kappa}_{j}^{\hat{\omega}_{j}}=\kappa_{j}^{\omega_{j}}$. Then $(20)$ reduces to $\left(\hat{\kappa}_{1}+s\right)^{\hat{\omega}_{1}}\left(\kappa_{j}+s\right)^{\omega_{j}}=\left(\hat{\kappa}_{j}+s\right)^{\omega_{j}}\left\{\left(\hat{\kappa}_{1}+s\right)^{\hat{\omega}_{1}}-\right.$ $\left.\hat{p}_{1} \hat{\kappa}_{1}^{\hat{\omega}_{1}}\right\}$. Setting $s=-\hat{\kappa}_{1}$ gives $\hat{p}_{1} \hat{\kappa}_{1}^{\hat{\omega}_{1}}\left(\hat{\kappa}_{j}-\hat{\kappa}_{1}\right)^{\omega_{j}}=0$, from which we deduce that $\hat{\kappa}_{j}=\hat{\kappa}_{1}$, and (20) further reduces to $\left(\kappa_{j}+s\right)^{\omega_{j}}=\left(\hat{\kappa}_{1}+s\right)^{\omega_{j}}-\hat{p}_{1} \hat{\kappa}_{1}^{\hat{\omega}_{1}}\left(\hat{\kappa}_{1}+s\right)^{-\hat{\omega}_{1}}$. Letting $s \rightarrow-\hat{\kappa}_{1}$, the left-hand side converges to $\left(\kappa_{j}-\hat{\kappa}_{1}\right)^{\omega_{j}}$, whereas the right-hand side diverges to $-\infty$. Hence, (20) has no admissible solutions.

Assume next that $p_{1} \in(0,1)$. If $\hat{p}_{1}=0$, a similar line of arguments shows that there are no admissible solutions. If $\hat{p}_{1} \in(0,1),(6)$ gives

$$
\frac{\left(1-\hat{p}_{1}\right)\left(\hat{\kappa}_{j} /\left(\hat{\kappa}_{j}+s\right)\right)^{\hat{\omega}_{j}}}{1-\hat{p}_{1}\left(\hat{\kappa}_{1} /\left(\hat{\kappa}_{1}+s\right)\right)^{\hat{\omega}_{1}}}=\frac{\left(1-p_{1}\right)\left(\kappa_{j} /\left(\kappa_{j}+s\right)\right)^{\omega_{j}}}{1-p_{1}\left(\kappa_{1} /\left(\kappa_{1}+s\right)\right)^{\omega_{1}}} .
$$

Rearranging the terms in the above identity leads to the equation

$$
\begin{aligned}
& \left(1-p_{1}\right) \kappa_{j}^{\omega_{j}}\left(\kappa_{1}+s\right)^{\omega_{1}}\left(\hat{\kappa}_{j}+s\right)^{\hat{\omega}_{j}}\left\{\left(\hat{\kappa}_{1}+s\right)^{\hat{\omega}_{1}}-\hat{p}_{1} \hat{\kappa}_{1}^{\hat{\omega}_{1}}\right\} \\
& \quad=\left(1-\hat{p}_{1}\right) \hat{\kappa}_{j}^{\hat{\omega}_{j}}\left(\hat{\kappa}_{1}+s\right)^{\hat{\omega}_{1}}\left(\kappa_{j}+s\right)^{\omega_{j}}\left\{\left(\kappa_{1}+s\right)^{\omega_{1}}-p_{1} \kappa_{1}^{\omega_{1}}\right\} .
\end{aligned}
$$

Divide both sides of $(21)$ by $\left(\kappa_{1}+s\right)^{\omega_{1}}\left(\hat{\kappa}_{1}+s\right)^{\hat{\omega}_{1}}\left(\hat{\kappa}_{j}+s\right)^{\hat{\omega}_{j}}$ and let $s \rightarrow \infty$. Then

$$
\left(1-p_{1}\right) \kappa_{j}^{\omega_{j}}=\left(1-\hat{p}_{1}\right) \hat{\kappa}_{j}^{\hat{\omega}_{j}} \lim _{s \rightarrow \infty}\left\{\frac{\left(\kappa_{j}+s\right)^{\omega_{j}}}{\left(\hat{\kappa}_{j}+s\right)^{\hat{\omega}_{j}}}-\frac{p_{1} \kappa_{1}^{\omega_{1}}\left(\kappa_{j}+s\right)^{\omega_{j}}}{\left(\hat{\kappa}_{j}+s\right)^{\hat{\omega}_{j}}\left(\kappa_{1}+s\right)^{\omega_{1}}}\right\} .
$$

In order for the right-hand side to converge to a constant, we must have $\omega_{j}=\hat{\omega}_{j}$, which implies that $\left(1-p_{1}\right) \kappa_{j}^{\omega_{j}}=\left(1-\hat{p}_{1}\right) \hat{\kappa}_{j}^{\hat{\omega}_{j}}$. Then $(21)$ reduces to

$$
\begin{aligned}
& \left(\kappa_{1}+s\right)^{\omega_{1}}\left(\hat{\kappa}_{j}+s\right)^{\omega_{j}}\left\{\left(\hat{\kappa}_{1}+s\right)^{\hat{\omega}_{1}}-\hat{p}_{1} \hat{\kappa}_{1}^{\hat{\omega}_{1}}\right\} \\
& \quad=\left(\hat{\kappa}_{1}+s\right)^{\hat{\omega}_{1}}\left(\kappa_{j}+s\right)^{\omega_{j}}\left\{\left(\kappa_{1}+s\right)^{\omega_{1}}-p_{1} \kappa_{1}^{\omega_{1}}\right\} .
\end{aligned}
$$


Setting $s=-\kappa_{1}$ gives $-p_{1} \kappa_{1}^{\omega_{1}}\left(\hat{\kappa}_{1}-\kappa_{1}\right)^{\hat{\omega}_{1}}\left(\kappa_{j}-\kappa_{1}\right)^{\omega_{j}}=0$, from which we deduce that either $\hat{\kappa}_{1}=\kappa_{1}$ or $\kappa_{j}=\kappa_{1}$. We study these two cases separately.

Case $(a): \hat{\kappa}_{1}=\kappa_{1}$. Assume first that $\omega_{1}>\hat{\omega}_{1}$. Equation (22) becomes

$$
\left(\kappa_{1}+s\right)^{\omega_{1}-\hat{\omega}_{1}}\left(\hat{\kappa}_{j}+s\right)^{\omega_{j}}\left\{\left(\kappa_{1}+s\right)^{\hat{\omega}_{1}}-\hat{p}_{1} \kappa_{1}^{\hat{\omega}_{1}}\right\}=\left(\kappa_{j}+s\right)^{\omega_{j}}\left\{\left(\kappa_{1}+s\right)^{\omega_{1}}-p_{1} \kappa_{1}^{\omega_{1}}\right\} .
$$

Setting $s=-\kappa_{1}$ gives $p_{1} \kappa_{1}^{\omega_{1}}\left(\kappa_{j}-\kappa_{1}\right)=0$. Hence, we must have $\kappa_{1}=\kappa_{j}$, and (23) becomes

$$
\left(\kappa_{1}+s\right)^{\omega_{1}-\hat{\omega}_{1}}\left(\hat{\kappa}_{j}+s\right)^{\omega_{j}}\left\{\left(\kappa_{1}+s\right)^{\hat{\omega}_{1}}-\hat{p}_{1} \kappa_{1}^{\hat{\omega}_{1}}\right\}=\left(\kappa_{1}+s\right)^{\omega_{j}}\left\{\left(\kappa_{1}+s\right)^{\omega_{1}}-p_{1} \kappa_{1}^{\omega_{1}}\right\} .
$$

We distinguish two sets of solutions.

(i) If $\hat{\kappa}_{j}=\kappa_{1},(24)$ reduces to $\left(\kappa_{1}+s\right)^{\omega_{1}-\hat{\omega}_{1}}\left\{\left(\kappa_{1}+s\right)^{\hat{\omega}_{1}}-\hat{p}_{1} \kappa_{1}^{\hat{\omega}_{1}}\right\}=\left\{\left(\kappa_{1}+s\right)^{\omega_{1}}-p_{1} \kappa_{1}^{\omega_{1}}\right\}$. Setting $s=-\kappa_{1}$ yields $p_{1} \kappa_{1}^{\omega_{1}}=0$, which is not admissible here because $p_{1} \kappa_{1}>0$.

(ii) If $\hat{\kappa}_{j} \neq \kappa_{1}$, dividing both sides of (24) by $\left(\kappa_{1}+s\right)^{\omega_{j}}$ and letting $s \rightarrow-\kappa_{1}$ entails that $\omega_{j}=$ $\omega_{1}-\hat{\omega}_{1}>0$, and $(24)$ reduces to $\left(\hat{\kappa}_{j}+s\right)^{\omega_{j}}\left\{\left(\kappa_{1}+s\right)^{\hat{\omega}_{1}}-\hat{p}_{1} \kappa_{1}^{\hat{\omega}_{1}}\right\}=\left(\kappa_{1}+s\right)^{\omega_{1}}-p_{1} \kappa_{1}^{\omega_{1}}$. Differentiating both sides of the equation with respect to $s$ gives $\omega_{j}\left(\hat{\kappa}_{j}+s\right)^{\omega_{j}-1}\left\{\left(\kappa_{1}+s\right)^{\hat{\omega}_{1}}-\hat{p}_{1} \kappa_{1}^{\hat{\omega}_{1}}\right\}+\hat{\omega}_{1}\left(\hat{\kappa}_{j}+s\right)^{\omega_{j}}\left(\kappa_{1}+s\right)^{\hat{\omega}_{1}-1}=\omega_{1}\left(\kappa_{1}+s\right)^{\omega_{1}-1}$. Letting $s \rightarrow-\hat{\kappa}_{j}$, the left-hand side of the equation converges to 0 if $\omega_{j}>1$ and diverges to $-\infty$ if $0<\omega_{j}<1$, whereas the right-hand side converges to $\omega_{1}\left(\kappa_{1}-\hat{\kappa}_{j}\right)^{\omega_{1}-1} \in$ $(0, \infty)$.

Hence, (22) has no admissible solutions in this case either. By using a similar line of arguments, we can show that (22) has no admissible solutions either when $\omega_{1}<\hat{\omega}_{1}$.

When $\hat{\omega}_{1}=\omega_{1},(22)$ reduces to

$$
\left(\hat{\kappa}_{j}+s\right)^{\omega_{j}}\left\{\left(\kappa_{1}+s\right)^{\omega_{1}}-\hat{p}_{1} \kappa_{1}^{\omega_{1}}\right\}=\left(\kappa_{j}+s\right)^{\omega_{j}}\left\{\left(\kappa_{1}+s\right)^{\omega_{1}}-p_{1} \kappa_{1}^{\omega_{1}}\right\} .
$$

If $\hat{\kappa}_{j} \neq \kappa_{j}$, setting $s=-\hat{\kappa}_{j}$ gives $\left(\kappa_{1}-\hat{\kappa}_{j}\right)^{\omega_{1}}=p_{1} \kappa_{1}^{\omega_{1}}$ and setting $s=-\kappa_{j}$ gives $\left(\kappa_{1}-\right.$ $\left.\kappa_{j}\right)^{\omega_{1}}=\hat{p}_{1} \kappa_{1}^{\omega_{1}}$. This implies that $\kappa_{1} \neq \hat{\kappa}_{j}$ and $\kappa_{1} \neq \kappa_{j}$. Taking the derivative with respect to $s$ on both sides of (25) yields

$$
\begin{aligned}
& \omega_{j}\left(\hat{\kappa}_{j}+s\right)^{\omega_{j}-1}\left\{\left(\kappa_{1}+s\right)^{\omega_{1}}-\left(\kappa_{1}-\kappa_{j}\right)^{\omega_{1}}\right\}+\omega_{1}\left(\hat{\kappa}_{j}+s\right)^{\omega_{j}}\left(\kappa_{1}+s\right)^{\omega_{1}-1} \\
& \quad=\omega_{j}\left(\kappa_{j}+s\right)^{\omega_{j}-1}\left\{\left(\kappa_{1}+s\right)^{\omega_{1}}-\left(\kappa_{1}-\hat{\kappa}_{j}\right)^{\omega_{1}}\right\}+\omega_{1}\left(\kappa_{j}+s\right)^{\omega_{j}}\left(\kappa_{1}+s\right)^{\omega_{1}-1} .
\end{aligned}
$$

As $s \rightarrow-\hat{\kappa}_{j}$, the left-hand side of (26) converges to 0 if $\omega_{j}>1$ and diverges to $-\infty$ if $0<\omega_{j}<1$, whereas the right-hand side converges to $\omega_{1}\left(\kappa_{j}-\hat{\kappa}_{j}\right)^{\omega_{j}}\left(\kappa_{1}-\hat{\kappa}_{j}\right)^{\omega_{1}-1} \in(0, \infty)$. Hence, (25) has no admissible solutions.

If $\hat{\kappa}_{j}=\kappa_{j}$ then $\left(\hat{\kappa}_{i}, \hat{\omega}_{i}\right)=\left(\kappa_{i}, \omega_{i}\right), i=1, j$. We also deduce from (25) that $\hat{p}_{1}=p_{1}$. Hence $\hat{p}_{j}=p_{j}$ using (19).

Case $(b): \hat{\kappa}_{1} \neq \kappa_{1}$ and $\kappa_{j}=\kappa_{1}$. Equation (22) reduces to

$$
\left(\kappa_{1}+s\right)^{\omega_{1}}\left(\hat{\kappa}_{j}+s\right)^{\omega_{j}}\left\{\left(\hat{\kappa}_{1}+s\right)^{\hat{\omega}_{1}}-\hat{p}_{1} \hat{\kappa}_{1}^{\hat{\omega}_{1}}\right\}=\left(\hat{\kappa}_{1}+s\right)^{\hat{\omega}_{1}}\left(\kappa_{1}+s\right)^{\omega_{j}}\left\{\left(\kappa_{1}+s\right)^{\omega_{1}}-p_{1} \kappa_{1}^{\omega_{1}}\right\} .
$$

Setting $s=-\hat{\kappa}_{1}$ gives $\hat{p}_{1} \hat{\kappa}_{1}^{\hat{\omega}_{1}}\left(\kappa_{1}-\hat{\kappa}_{1}\right)^{\omega_{1}}\left(\hat{\kappa}_{j}-\hat{\kappa}_{1}\right)^{\omega_{j}}=0$. Because $\hat{\kappa}_{1} \neq \kappa_{1}$, we must have $\hat{\kappa}_{1}=\hat{\kappa}_{j}$, and (27) reduces to

$$
\left(\kappa_{1}+s\right)^{\omega_{1}-\omega_{j}}\left\{\left(\hat{\kappa}_{1}+s\right)^{\hat{\omega}_{1}}-\hat{p}_{1} \hat{\kappa}_{1}^{\hat{\omega}_{1}}\right\}=\left(\hat{\kappa}_{1}+s\right)^{\hat{\omega}_{1}-\omega_{j}}\left\{\left(\kappa_{1}+s\right)^{\omega_{1}}-p_{1} \kappa_{1}^{\omega_{1}}\right\} .
$$

We consider the following cases separately.

(i) If $\omega_{1}>\omega_{j}$, setting $s=-\kappa_{1}$ yields $p_{1} \kappa_{1}^{\omega_{1}}\left(\hat{\kappa}_{1}-\kappa_{1}\right)^{\hat{\omega}_{1}-\omega_{j}}=0$, which has no admissible solutions. 
(ii) If $\hat{\omega}_{1}>\omega_{j}$, setting $s=-\hat{\kappa}_{1}$ yields $\hat{p}_{1} \hat{\kappa}_{1}^{\hat{\omega}_{1}}\left(\kappa_{1}-\hat{\kappa}_{1}\right)^{\omega_{1}-\omega_{j}}=0$, which has no admissible solutions.

(iii) If $\omega_{j}>\omega_{1}$ and $\omega_{j}>\hat{\omega}_{1},(28)$ can be rewritten as

$$
\left(\hat{\kappa}_{1}+s\right)^{\omega_{j}-\hat{\omega}_{1}}\left\{\left(\hat{\kappa}_{1}+s\right)^{\hat{\omega}_{1}}-\hat{p}_{1} \hat{\kappa}_{1}^{\hat{\omega}_{1}}\right\}=\left(\kappa_{1}+s\right)^{\omega_{j}-\omega_{1}}\left\{\left(\kappa_{1}+s\right)^{\omega_{1}}-p_{1} \kappa_{1}^{\omega_{1}}\right\} .
$$

Setting $s=-\hat{\kappa}_{1}$ yields $\left(\kappa_{1}-\hat{\kappa}_{1}\right)^{\omega_{1}}=p_{1} \kappa_{1}^{\omega_{1}}$, and setting $s=-\kappa_{1}$ yields $\left(\hat{\kappa}_{1}-\kappa_{1}\right)^{\hat{\omega}_{1}}=$ $\hat{p}_{1} \hat{\kappa}_{1}^{\hat{\omega}_{1}}$. Differentiating (29) with respect to $s$ gives

$$
\begin{aligned}
& \left(\omega_{j}-\hat{\omega}_{1}\right)\left(\hat{\kappa}_{1}+s\right)^{\omega_{j}-\hat{\omega}_{1}-1}\left\{\left(\hat{\kappa}_{1}+s\right)^{\hat{\omega}_{1}}-\left(\hat{\kappa}_{1}-\kappa_{1}\right)^{\hat{\omega}_{1}}\right\}+\hat{\omega}_{1}\left(\hat{\kappa}_{1}+s\right)^{\omega_{j}-1} \\
& \quad=\left(\omega_{j}-\omega_{1}\right)\left(\kappa_{1}+s\right)^{\omega_{j}-\omega_{1}-1}\left\{\left(\kappa_{1}+s\right)^{\omega_{1}}-\left(\kappa_{1}-\hat{\kappa}_{1}\right)^{\omega_{1}}\right\}+\omega_{1}\left(\kappa_{1}+s\right)^{\omega_{j}-1} .
\end{aligned}
$$

Letting $s \rightarrow-\hat{\kappa}_{1}$, the left-hand side of the equation either converges to 0 (if $\omega_{j}-\hat{\omega}_{1}>1$ ) or diverges to $-\infty$ (if $\omega_{j}-\hat{\omega}_{1}<1$ ), whereas the right-hand side converges to $\omega_{1}\left(\kappa_{1}-\right.$ $\left.\hat{\kappa}_{1}\right)^{\omega_{j}-1} \in(0, \infty)$. Hence, (29) has no admissible solutions.

(iv) If $\omega_{j}=\omega_{1}$ and $\omega_{j}>\hat{\omega}_{1},(28)$ can be rewritten as

$$
\left(\hat{\kappa}_{1}+s\right)^{\omega_{j}-\hat{\omega}_{1}}\left\{\left(\hat{\kappa}_{1}+s\right)^{\hat{\omega}_{1}}-\hat{p}_{1} \hat{\kappa}_{1}^{\hat{\omega}_{1}}\right\}=\left\{\left(\kappa_{1}+s\right)^{\omega_{1}}-p_{1} \kappa_{1}^{\omega_{1}}\right\} .
$$

Taking the derivative with respect to $s$ on both sides of (30) gives

$$
\left(\omega_{j}-\hat{\omega}_{1}\right)\left(\hat{\kappa}_{1}+s\right)^{\omega_{j}-\hat{\omega}_{1}-1}\left\{\left(\hat{\kappa}_{1}+s\right)^{\hat{\omega}_{1}}-\hat{p}_{1} \hat{\kappa}_{1}^{\hat{\omega}_{1}}\right\}+\hat{\omega}_{1}\left(\hat{\kappa}_{1}+s\right)^{\omega_{j}-1}=\omega_{1}\left(\kappa_{1}+s\right)^{\omega_{1}-1} .
$$

Letting $s \rightarrow-\hat{\kappa}_{1}$, the left-hand side of the equation either converges to 0 (if $\omega_{j}-\hat{\omega}_{1}>1$ ) or diverges to $-\infty$ (if $\omega_{j}-\hat{\omega}_{1}<1$ ), whereas the right-hand side converges to $\omega_{1}\left(\kappa_{1}-\right.$ $\left.\hat{\kappa}_{1}\right)^{\omega_{j}-1} \in(0, \infty)$. Hence, $(30)$ has no admissible solutions. The case in which $\omega_{j}>\omega_{1}$ and $\omega_{j}=\hat{\omega}_{1}$ is handled similarly, and has no solutions either.

(v) If $\omega_{j}=\omega_{1}$ and $\omega_{j}=\hat{\omega}_{1},(28)$ reduces to $\left(\hat{\kappa}_{1}+s\right)^{\omega_{1}}-\hat{p}_{1} \hat{\kappa}_{1}^{\omega_{1}}=\left(\kappa_{1}+s\right)^{\omega_{1}}-p_{1} \kappa_{1}^{\omega_{1}}$, which has no admissible solutions because $\hat{\kappa}_{1} \neq \kappa_{1}$.

\subsection{A Smith-Martin process}

We consider a generalization of the Smith-Martin model originally proposed in [8]. The process assumes that, conditional on $\xi=j, j \in \mathcal{g}^{*}(p)$, the lifespan takes the form $\tau=\tau_{A_{j}}+\delta_{j}$, where $\tau_{A_{j}}$ follows an exponential distribution with parameter $\psi_{j}$, and where $\delta_{j}$ is a nonnegative constant. In the original formulation of the model, $\tau_{A_{2}}$ essentially represents the duration spent by the cell in the $G_{0} / G_{1}$ phases, and $\delta_{2}$ is the time spent by the cell in the $S, G_{2}$, and $M$ (and part of $\left.G_{1}\right)$ phases. Here $\mathcal{L}_{g_{j}}(s)=\mathrm{e}^{-\delta_{j} s} \psi_{j} /\left(\psi_{j}+s\right)$, where it can be extended to $s \in \mathbb{R} \backslash\left\{-\psi_{j}\right\}$ by analytic continuation. This process is identical to the process of Section 3.1 if $\delta_{j}=0, j \in \mathcal{g}^{*}(p)$. Let $\mathcal{M}^{\Im \mathcal{M}}$ denote the family of Smith-Martin processes. Write $\mathcal{C}_{p, G}^{\mathcal{S M}}=\mathcal{C}_{p, G} \cap \mathcal{M}^{\mathcal{S M}}$ for the class of Smith-Martin processes equivalent to the process with characteristics $(p, G)$. This process is not always identifiable.

Corollary 3. Suppose that $J=2$ and, for every $j \in \mathcal{F}^{*}(p)$, that $G_{j}(t)=1-\mathrm{e}^{-\psi_{j}\left(t-\delta_{j}\right)}$ $\left(t \geq \delta_{j}\right)$. Then $(p, G)$ is uniquely identified by $\{Z(t), t \geq 0\}$ except in the following cases.

Case 1. If $\psi_{j}=\psi, j \in \mathcal{g}^{*}(p)$, and $\delta_{1}=0$ when $p_{1}=0$ (Bellman-Harris case), $\mathcal{C}_{p, G}^{\& \mathcal{M}}$ includes the Smith-Martin processes with characteristics $(\hat{p}, \hat{G}) \in\left\{\hat{p}_{1} \in(0,1), \hat{p}_{j}\right.$, $p_{j}\left(1-\hat{p}_{1}\right) /\left(1-p_{1}\right), \hat{\delta}_{1}=0, \hat{\delta}_{j}=\delta_{j}, \hat{\psi}_{1}=\hat{\psi}_{j}=\psi\left(1-p_{1}\right) /\left(1-\hat{p}_{1}\right), j \in$ $\left.\mathcal{g}^{*}(p) \backslash\{1\}\right\} \cup\left\{\hat{p}_{1}=0, \hat{p}_{j}=p_{j} /\left(1-p_{1}\right), \hat{\delta}_{j}=\delta_{j}, \hat{\psi}_{j}=\psi\left(1-p_{1}\right), j \in \mathcal{g}^{*}(p) \backslash\{1\}\right\}$. 
Case 2. If $p_{1} \in(0,1), p_{1} \neq 1-\psi / \psi_{1}, \delta_{1}=0, \psi_{j}=\psi, j \in \mathcal{G}^{*}(p) \backslash\{1\}$, and $\psi<\psi_{1}$ ('extended' Bellman-Harris case), $e_{p, G}^{8 \mathcal{M}}$ consists of the Smith-Martin processes with characteristics $(p, G)$ and $(\hat{p}, \hat{G})$, where $\hat{p}_{1}=1-\psi / \psi_{1}, \hat{p}_{j}=p_{j} \psi /\left\{\left(1-p_{1}\right) \psi_{1}\right\}$, $\hat{\delta}_{1}=0, \hat{\delta}_{j}=\delta_{j}, \hat{\psi}_{1}=\psi_{1}$, and $\hat{\psi}_{j}=\left(1-p_{1}\right) \psi_{1}, j \in\{0,2\}$.

Proof. Let $(\hat{p}, \hat{G})$ denote the characteristics of any process in $\mathcal{C}_{p, G}$. Assume first that $p_{1}=0$. If $\hat{p}_{1}=0$, Lemma 1 yields $(\hat{p}, \hat{G})=(p, G)$. If $\hat{p}_{1} \in(0,1),(7)$ gives

$$
\left(1-\hat{p}_{1}\right) \frac{\mathrm{e}^{-\hat{\delta}_{j} s} \hat{\psi}_{j}}{\hat{\psi}_{j}+s}=\frac{\mathrm{e}^{-\delta_{j} s} \psi_{j}}{\psi_{j}+s}\left\{1-\hat{p}_{1} \frac{\mathrm{e}^{-\hat{\delta}_{1} s} \hat{\psi}_{1}}{\hat{\psi}_{1}+s}\right\}
$$

Taking the logarithm of both sides of the equation, we obtain

$$
-\hat{\delta}_{j} s+\log \hat{\psi}_{j}\left(1-\hat{p}_{1}\right)-\log \left(\hat{\psi}_{j}+s\right)=-\delta_{j} s-\log \left(\psi_{j}+s\right)+\log \psi_{j}\left(1-\hat{p}_{1} \frac{\mathrm{e}^{-\hat{\delta}_{1} s} \hat{\psi}_{1}}{\hat{\psi}_{1}+s}\right) .
$$

Dividing both sides of the equation by $s$ and letting $s \rightarrow \infty$ yields $\hat{\delta}_{j}=\delta_{j}$, and (31) reduces to $\left(1-\hat{p}_{1}\right) \hat{\psi}_{j}\left(\psi_{j}+s\right)\left(\hat{\psi}_{1}+s\right)-\psi_{j}\left(\hat{\psi}_{j}+s\right)\left(\hat{\psi}_{1}+s\right)=\psi_{j}\left(\hat{\psi}_{j}+s\right) \hat{p}_{1} \hat{\psi}_{1} \mathrm{e}^{-\hat{\delta}_{1} s}$. Taking again the logarithm of both sides of the equation, dividing by $s$, and letting $s \rightarrow \infty$ yields $\hat{\delta}_{1}=0$. Hence, (31) leads to (18), from which we deduce that $\hat{\psi}_{1}=\hat{\psi}_{j}=\psi /\left(1-\hat{p}_{1}\right)$, where $\psi:=$ $\psi_{j}, j \in \mathcal{G}^{*}(p)$, and $\hat{p}_{j}=p_{j}\left(1-\hat{p}_{1}\right), \hat{p}_{1} \in(0,1)$. This proves part of case 1 of Corollary 3 .

Assume next that $p_{1} \in(0,1)$. If $\hat{p}_{1}=0$, the same line of arguments applies, and, by symmetry, we find that the process with characteristics $(\hat{p}, \hat{G})$ satisfying $\hat{\delta}_{j}=\delta_{j}, \hat{\psi}_{j}=\psi(1-$ $\left.p_{1}\right)$, and $\hat{p}_{j}=p_{j} /\left(1-p_{1}\right), j \in \mathcal{G}^{*}(p) \backslash\{1\}$, belongs to $\mathcal{C}_{p, G}^{\mathcal{S M}}$ if $\psi_{j}=\psi, j \in \mathcal{F}^{*}(p)$, and $\delta_{1}=0$. This also proves part of case 1 .

Assume now that $p_{1} \in(0,1)$ and $\hat{p}_{1} \in(0,1)$. Then, for every $j \in \mathcal{g}^{*}(p) \backslash\{1\},(6)$ gives

$$
\left(1-\hat{p}_{1}\right) \frac{\mathrm{e}^{-\hat{\delta}_{j} s} \hat{\psi}_{j}}{\hat{\psi}_{j}+s}\left\{1-p_{1} \frac{\mathrm{e}^{-\delta_{1} s} \psi_{1}}{\psi_{1}+s}\right\}=\left(1-p_{1}\right) \frac{\mathrm{e}^{-\delta_{j} s} \psi_{j}}{\psi_{j}+s}\left\{1-\hat{p}_{1} \frac{\mathrm{e}^{-\hat{\delta}_{1} s} \hat{\psi}_{1}}{\hat{\psi}_{1}+s}\right\} \text {. }
$$

Taking the logarithm, dividing both sides of (32) by $s$, and letting $s \rightarrow \infty$ implies that $\hat{\delta}_{j}=\delta_{j}$, and $(32)$ reduces to

$$
\left(1-\hat{p}_{1}\right) \frac{\hat{\psi}_{j}}{\hat{\psi}_{j}+s}\left\{1-p_{1} \frac{\mathrm{e}^{-\delta_{1} s} \psi_{1}}{\psi_{1}+s}\right\}=\left(1-p_{1}\right) \frac{\psi_{j}}{\psi_{j}+s}\left\{1-\hat{p}_{1} \frac{\mathrm{e}^{-\hat{\delta}_{1} s} \hat{\psi}_{1}}{\hat{\psi}_{1}+s}\right\} .
$$

Multiplying both sides by $s$ and letting $s \rightarrow \infty$, we obtain $\left(1-\hat{p}_{1}\right) \hat{\psi}_{j}=\left(1-p_{1}\right) \psi_{j}$. Then (32) becomes

$$
\left(\psi_{j}-\hat{\psi}_{j}\right)\left(\psi_{1}+s\right)\left(\hat{\psi}_{1}+s\right)=p_{1} \psi_{1} \mathrm{e}^{-\delta_{1} s}\left(\psi_{j}+s\right)\left(\hat{\psi}_{1}+s\right)-\hat{p}_{1} \hat{\psi}_{1} \mathrm{e}^{-\hat{\delta}_{1} s}\left(\hat{\psi}_{j}+s\right)\left(\psi_{1}+s\right) .
$$

We distinguish four sets of solutions.

(i) If $\delta_{1}>0$ and $\hat{\delta}_{1}>0$, dividing both sides of (33) by $s^{2}$ and letting $s \rightarrow \infty$ yields $\hat{\psi}_{j}=\psi_{j}$. Then, (33) reduces to $p_{1} \psi_{1} \mathrm{e}^{-\delta_{1} s}\left(\hat{\psi}_{1}+s\right)=\hat{p}_{1} \hat{\psi}_{1} \mathrm{e}^{-\hat{\delta}_{1} s}\left(\psi_{1}+s\right)$, from which we deduce that $\hat{\delta}_{1}=\delta_{1}, \hat{p}_{1}=p_{1}$, and $\hat{\psi}_{1}=\psi_{1}$. Hence, $(\hat{p}, \hat{G})=(p, G)$.

(ii) If $\delta_{1}>0$ and $\hat{\delta}_{1}=0$, rearranging the terms of (33) leads to $\left(\psi_{1}+s\right)\left\{\left(\psi_{j}-\hat{\psi}_{j}\right)\left(\hat{\psi}_{1}+s\right)+\right.$ $\left.\hat{p}_{1} \hat{\psi}_{1}\left(\hat{\psi}_{j}+s\right)\right\}=p_{1} \psi_{1} \mathrm{e}^{-\delta_{1} s}\left(\psi_{j}+s\right)\left(\hat{\psi}_{1}+s\right)$. Letting $s \rightarrow \infty$, the left-hand side of the equation diverges to infinity, whereas the right-hand side converges to 0. Hence, (33) has no admissible solutions. 
(iii) If $\delta_{1}=0$ and $\hat{\delta}_{1}>0$, a similar line of arguments shows that (33) has no admissible solutions.

(iv) If $\delta_{1}=0$ and $\hat{\delta}_{1}=0,(33)$ is equivalent to (19). The values of $\hat{p}$ and $\hat{\psi}_{j}, j \in g^{*}(\hat{p})$ that solve the equation are given in Corollary 1 , and lead to parts of case 1 and case 2 .

\section{Acknowledgements}

The research of the second author was supported by NIH R01 grants NS039511, CA134839, and AI069351.

\section{References}

[1] Gyllenberg, M. and Webb, G. F. (1990). A nonlinear structured population model of tumor growth with quiescence. J. Math. Biol. 28, 671-694.

[2] Haccou, P., Jagers, P. And Vatutin, V. A. (2007). Branching Processes: Variation, Growth, and Extinction of Populations. Cambridge University Press.

[3] Hyrien, O., Mayer-Pröschel, M., Noble, M. and Yakovlev, A. (2005). A stochastic model to analyze clonal data on multi-type cell populations. Biometrics 61, 199-207.

[4] JAGERS, P. (1975). Branching Processes with Biological Applications. John Wiley, London.

[5] Kimmel, M. And Axelrod, D. E. (2002). Branching Processes in Biology. Springer, New York.

[6] ReDner, R. (1981). Note on the consistency of the maximum likelihood estimate for nonidentifiable distributions. Ann. Statist. 9, 225-228.

[7] Sevastyanov, B. A. (1971). Branching Processes. Nauka, Moscow (in Russian).

[8] Smith, J. A. and Martin, L. (1973). Do cells cycle? Proc. Nat. Acad. Sci. 70, 1263-1267.

[9] Yakovlev, A. Y. and Yanev, N. M. (1989). Transient Processes in Cell Proliferation Kinetics. Springer, Berlin. 